\title{
A Case Report of an Emergency Hypertensive Episode During Trastuzumab Therapy
}

\author{
Adriano Pacheco Mendes ${ }^{1}$, Jerina Nogueira ${ }^{1}$, André Mendes ${ }^{1}$, Joana Cochicho ${ }^{1}$, Isabel Lavadinho ${ }^{1}$ \\ 1. Internal Medicine Department, Hospital Dr. José Maria Grande - Unidade Local de Saúde do Norte Alentejano, \\ Portalegre, PRT
}

Corresponding author: Adriano Pacheco Mendes, apachecomendes@gmail.com

\begin{abstract}
Human epidermal growth factor receptor 2 (HER2+) targeted therapies, such as trastuzumab emtansine, has been shown to have a significant positive effect on the outcome of breast cancer. Rarely, this therapy can cause adverse cardiovascular effects such as cardiac dysfunction, arrhythmias and hypertension. We report a case of a 60-year-old woman who presented to the emergency department with an episode of hypertensive emergency. She had been recently diagnosed with hypertension which was found to be poorly controlled. The recent administration of trastuzumab together with this hypertensive episode led us to suspect trastuzumab as the cause for this rise in her blood pressure. With this case we intend to raise awareness of hypertension, a potentially preventable condition, as an adverse effect of human epidermal growth factor receptor 2 targeted therapies such as trastuzumab. Additionally we propose an appropriate and careful management of arterial hypertension among those receiving this first-in-class drug.
\end{abstract}

Categories: Cardiology, Emergency Medicine, Internal Medicine

Keywords: trastuzumab, breast cancer, hypertension, intracerebral hemorrhage, cardio-oncology

\section{Introduction}

Human epidermal growth factor receptor 2 (HER2+) targeted therapies, such as trastuzumab, have improved significantly the management of breast cancer and its outcome [1-2].

Trastuzumab is a life-extending therapy for breast cancer patients overexpressing HER2+, despite having a known cardiotoxic risk and an increased risk for cardiovascular events [3-5]. As a primary clinical cardiovascular side effect seen with HER2+ targeted therapies, cardiac dysfunction is manifested by an asymptomatic decrease in left ventricular ejection fraction. Less commonly, developing hypertension may be seen as a secondary effect which is not totally understood [6]. If uncontrolled, systemic arterial hypertension can lead to major events such as hypertensive emergencies [7].

Review began 07/09/2021 Review ended 07/28/2021 Published 08/09/2021

๑) Copyright 2021

Pacheco Mendes et al. This is an open access article distributed under the terms of the Creative Commons Attribution License CC-BY 4.0., which permits unrestricted use, distribution, and reproduction in any medium, provided the original author and source are credited.

\section{Case Presentation}

We report the case of a 60-year-old woman admitted to the emergency department following a minor road traffic accident. The patient had a medical history of breast infiltrating ductal carcinoma (HER2+) under trastuzumab monotherapy for seven months and had been submitted to chemotherapy with FEC regimen (5-fluorouracil, epidoxorubicin and cyclophosphamide) four years before trastuzumab initiation. The patient had recently been diagnosed (six weeks before) with hypertension and started treatment with an angiotensin-converting enzyme inhibitor (enalapril $10 \mathrm{mg}$ once daily) together with a calcium channel blocker (amlodipine $5 \mathrm{mg}$ once daily). Despite treatment, it was still poorly controlled.

On examination in the emergency room, there was no evidence of traumatic lesions in keeping with the paramedics' initial report, the patient was hypertensive (196/97 $\mathrm{mmHg}$ ), with a Glasgow Coma Scale score of 14 (E4V4M6), following verbal commands but with a new onset left hemiplegia with a National Institutes of Health Stroke Scale (NIHSS) score of 6 (4 points on left arm motor drift and 2 points on left leg motor drift). A non-enhanced computed tomography of the head revealed a right intracerebral haemorrhage of 70 $\mathrm{x} 40 \mathrm{~mm}$ with associated midline shift of $4 \mathrm{~mm}$ to the left side (Figure 1). 


\section{Cureus}

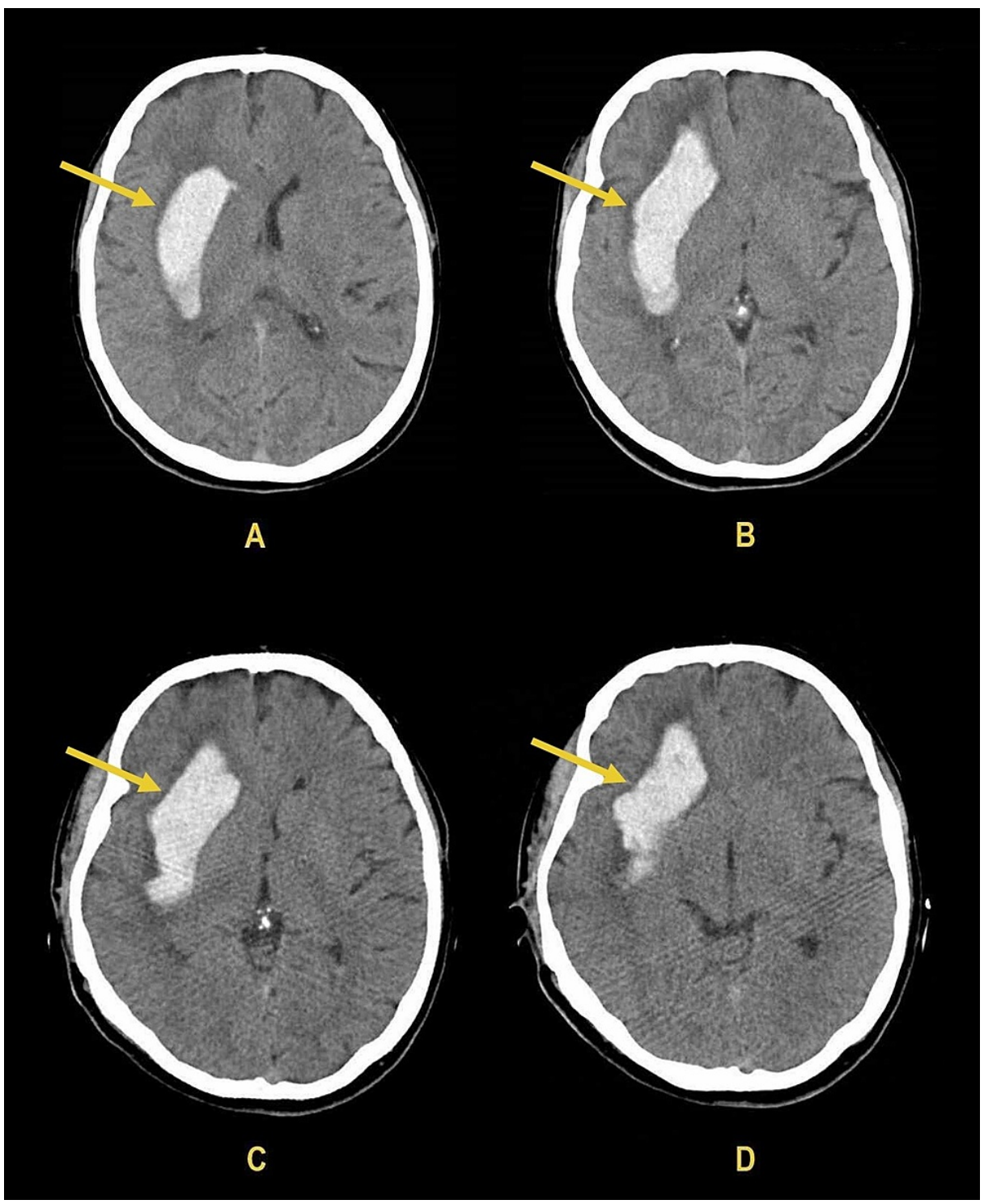

FIGURE 1: Intracerebral haemorrhage with associated midline shift of 4 $\mathrm{mm}$ to the left side on computed tomography scan of the head

She was diagnosed with a spontaneous haemorrhagic stroke secondary to uncontrolled blood pressure. Other investigations, such as electrocardiogram and blood analysis, did not show any significant abnormalities. With regards to the transthoracic echocardiogram, it was found to have a segmental wall motion abnormality with a left ventricular mid inferior and basal inferior wall hypokinesia with no compromise of ventricular systolic function. We did not have access to previous echocardiograms for comparison but according to patient's verbal information, there were no abnormalities on the echocardiography examination performed prior to trastuzumab initiation. Despite information provided by the patient with regards to her medical history, we were unable to safely exclude that the left ventricular regional wall motion abnormality was secondary to the previous chemotherapy regimen or due to a different etiology.

Even though the patient presented with an intracerebral haemorrhage which could lead to a poor outcome, recovery was favourable with minor neurological deficits (NIHSS score of 1 on left arm motor drift) after 10 days of hospitalization. Short follow-up appointment with her oncologist was set up and antihypertensive therapy optimized before discharge (enalapril $20 \mathrm{mg}$ once daily, amlodipine $10 \mathrm{mg}$ once daily and indapamide $2.5 \mathrm{mg}$ once daily).

\section{Discussion}

As medicine takes further steps towards the development of new treatment strategies, more women are surviving and living with breast cancer. Despite the significant improvements in outcomes for women with HER2-positive tumours, breast cancer patients are regarded as a population with high cardiovascular risk 
[8]. This risk is higher when associated with trastuzumab treatment due to the known adverse effects which increases incidence of cardiovascular conditions such as left ventricular dysfunction, arrhythmias, hypertension and cardiomyopathy $[4,9]$.

Systemic arterial hypertension is described as an uncommon adverse effect [7,10]. Although it could be coincidental, according to the patient's past medical history, there was a strong temporal association between the onset of arterial hypertension and the timing of initiation of trastuzumab, which she had been taking for the previous seven months, suggesting that it was likely related to trastuzumab exposure. No other evidence pointed to an alternative cause of secondary hypertension.

Identifying risk factors associated with trastuzumab-induced cardiotoxicity allows more targeted and intensive screening for at-risk patients. Since hypertension is a potentially preventable condition during breast cancer treatment and one of the most consistent predictors of trastuzumab-

mediated cardiotoxicity, a more regular routine cardiac monitoring is desirable. In addition, surveillance and management of hypertension and other cardiovascular risk factors should be routinely performed during trastuzumab treatment in order to avoid events like hypertensive emergencies and their complications $[9,11]$.

\section{Conclusions}

Given the adverse outcomes associated with uncontrolled hypertension and the prospect of increasing use of trastuzumab for patients with metastatic breast cancer, we attempt to raise awareness to hypertension, a potentially preventable risk factor, as a possible uncommon adverse effect of humanized anti-HER2 positive monoclonal antibodies such as trastuzumab. We also intend to propose an appropriate and careful management of any secondary arterial hypertension incidence among those receiving this first-inclass drug, which continues to have a favourable benefit-risk ratio.

\section{Additional Information \\ Disclosures}

Human subjects: Consent was obtained or waived by all participants in this study. Conflicts of interest: In compliance with the ICMJE uniform disclosure form, all authors declare the following: Payment/services info: All authors have declared that no financial support was received from any organization for the submitted work. Financial relationships: All authors have declared that they have no financial relationships at present or within the previous three years with any organizations that might have an interest in the submitted work. Other relationships: All authors have declared that there are no other relationships or activities that could appear to have influenced the submitted work.

\section{References}

1. Cameron D, Piccart-Gebhart MJ, Gelber RD, et al.: 11 years' follow-up of trastuzumab after adjuvant chemotherapy in HER2-positive early breast cancer: final analysis of the HERceptin Adjuvant (HERA) trial. Lancet. 2017, 389:1195-1205. 10.1016/S0140-6736(16)32616-2

2. Wildiers H, Tryfonidis K, Dal Lago L, et al.: Pertuzumab and trastuzumab with or without metronomic chemotherapy for older patients with HER2-positive metastatic breast cancer (EORTC 75111-10114): an open-label, randomised, phase 2 trial from the Elderly Task Force/Breast Cancer Group. Lancet Oncol. 2018, 19:323-336. 10.1016/S1470-2045(18)30083-4

3. Morash M, Mitchell H, Yu A, Campion T, Beltran H, Elemento O, Pathak J: CATCH-KB: establishing a pharmacogenomics variant repository for chemotherapy-induced cardiotoxicity. AMIA Jt Summits Transl Sci Proc. 2018, 2017:168-177.

4. Herrmann J, Yang EH, Iliescu CA, et al.: Vascular toxicities of cancer therapies: the old and the new--An evolving avenue. Circulation. 2016, 133:1272-1289. 10.1161/CIRCULATIONAHA.115.018347

5. Ewer MS, Ewer SM: Cardiotoxicity of anticancer treatments: what the cardiologist needs to know . Nat Rev Cardiol. 2010, 7:564-575. 10.1038/nrcardio.2010.121

6. Herrmann J, Herrmann SM, Haddad TC: New-onset heart failure in association with severe hypertension during trastuzumab therapy. Mayo Clin Proc. 2014, 89:1734-1739. 10.1016/j.mayocp.2014.08.011

7. U.S. Food and Drug Administration. Herceptin (trastuzumab). (2010). Accessed: December 21, 2020 : https://www.accessdata.fda.gov/drugsatfda_docs/label/2010/103792s5256lbl.pdf.

8. Mehta LS, Watson KE, Barac A, et al.: Cardiovascular disease and breast cancer: where these entities intersect: a scientific statement from the American Heart Association. Circulation. 2018, 137:30-66. 10.1161/CIR.0000000000000556

9. Piotrowski G, Gawor R, Stasiak A, Gawor Z, Potemski P, Banach M: Cardiac complications associated with trastuzumab in the setting of adjuvant chemotherapy for breast cancer overexpressing human epidermal growth factor receptor type 2 - a prospective study. Arch Med Sci. 2012, 8:227-235. 10.5114/aoms.2012.28549

10. von Minckwitz G, Huang CS, Mano MS, et al.: Trastuzumab emtansine for residual invasive HER2-positive breast cancer. N Engl J Med. 2019, 380:617-628. 10.1056/NEJMoa1814017

11. Chen MF, Manson DK, Yuan A, Crew KD: Trastuzumab-induced cardiotoxicity and hypertension among racially and ethnically diverse patients with HER2-positive early-stage breast cancer. J Clin Oncol. 2020, 38:10.1200/JCO.2020.38.15_suppl.e24092 